\title{
Understanding the Effect of Additives in Li-ion and Li-Sulfur Batteries by Operando ec- (S)TEM
}

\author{
B. Layla Mehdi ${ }^{1,2}$, Andrew Stevens ${ }^{3}$, Jiangfeng Qian ${ }^{1,4}$, Chiwoo Park ${ }^{5}$, Wu Xu ${ }^{1,4}$, Wesley A. \\ Henderson $^{4}$, Ji-Guang Zhang ${ }^{1,4}$, Karl T. Mueller ${ }^{1,6,7}$ and Nigel D. Browning ${ }^{1,2,8}$ \\ 1. Joint Center for Energy Storage Research (JCESR), Pacific Northwest National Laboratory (PNNL), \\ Richland, WA 99352, USA. \\ 2. Physical and Computational Science Directorate, PNNL, Richland, WA 99352, USA. \\ 3. National Security Directorate, PNNL, Richland, WA 99352, USA. \\ 4. Energy and Environmental Directorate, PNNL, Richland, WA 99352, USA. \\ 5. Industrial and Manufacturing Engineering, Florida State University, Tallahassee, FL 32306, USA. \\ 6. Environmental Molecular Sciences Laboratory, PNNL, Richland, WA 99352, USA. \\ 7. Department of Chemistry, Penn State University, University Park, PA, 16802, USA. \\ 8. Materials Science and Engineering, University of Washington, Seattle, WA 98195, USA.
}

Lithium (Li)-Sulfur (S) batteries are currently one of the most attractive battery systems for high capacity energy storage applications, potentially meeting a large demand by giving 3-5 fold higher energy densities (from $2600 \mathrm{Wh} \mathrm{kg}^{-1}$ to $2800 \mathrm{Wh} \mathrm{kg}^{-1}$ ) than state-of-the-art Li-ion batteries [1]. Additionally, elemental sulfur is cheap, safe, nontoxic and highly abundant. However, the practical application of a Li-sulfur batteries is still challenging due to the insulating nature of elemental sulfur cathode, dissolution of intermediate discharge products (polysulfides, $\mathrm{Li}_{2} \mathrm{~S}_{\mathrm{n}}, 3 \leq \mathrm{n} \leq 6$ ) into the electrolyte and the "shuttle effect" which leads to reduced Columbic efficiency in the charging stage, and the formation of a passivating Solid Electrolyte Interphase (SEI) layer at the Li anode contributing to the active mass loss available for discharge (self-discharge) [2]. These multi-step electrochemical reduction and oxidation reactions involving polysulfides are very complex and require an increased fundamental understanding to bring Li-S technology to wide-spread commercialization. There are many strategies to improve the interfacial stability of the $\mathrm{Li}$ anode and control/suppress $\mathrm{Li}$ dendrite growth, which is highly dependent on nature of electrolyte itself, such as mixture of different electrolyte solvents, salts and additives (e.g. $\mathrm{HF}, \mathrm{LiNO}_{3}$ etc).

Here we use an operando electrochemical cell (ec-cell) [3] in the scanning transmission electron microscope (STEM) to investigate the role and mechanism of electrolyte additives and the initial stages of $\mathrm{Li}$ deposition/dissolution and formation of the SEI layer. As a test of the fundamental process, we compare two commercially used electrolytes, lithium hexafluorophosphate $\left(\mathrm{LiPF}_{6}\right)$ in propylene carbonate (PC) electrolyte with controlled trace-amounts of water (10 ppm and $50 \mathrm{ppm})$ and LiTFSI in DOL/DME with $\mathrm{LiNO}_{3}$ to develop a mechanism for smooth SEI layer formation in Li-ion and Li-sulfur batteries. Figure 1A shows a comparison of the two cyclic voltammograms of electrolyte with $10 \mathrm{ppm}$ and $50 \mathrm{ppm}$ of water on $\mathrm{Li}$ dendrite deposition/dissolution and Figure 1B shows the corresponding quantified amount of $\mathrm{Li}$ that has been deposited and stripped during these cycles (extracted from the movies with a tracing algorithm). Figures 1C-D show bright field (BF) STEM images of Li metal deposits at the interface between the Pt working electrode in the presence of $10 \mathrm{ppm}$ (Figure 1C) and 50 ppm (Figure 1D) in $\mathrm{LiPF}_{6}$ in $\mathrm{PC}$ electrolyte. The higher concentration of water, $50 \mathrm{ppm}$, leads to increased concentration of $\mathrm{HF}$ additive and larger grain size growth through increased diffusion of $\mathrm{Li}$ ions across the SEI layer during battery cycling. Figure 1E-F shows a scanning electron microscopy (SEM) images of Li dendrites deposited on a $\mathrm{Cu}$ electrode surface in the presence of $10 \mathrm{ppm}$ (Figure 1E) 
and $50 \mathrm{ppm}$ (Figure $1 \mathrm{~F}$ ) of water at the current densities of $1 \mathrm{~mA} / \mathrm{cm}^{2}$ for $15 \mathrm{~h}$. The Li dendrite in the electrolyte contacting $10 \mathrm{ppm}$ shows a typical dendritic microstructure. However, the electrolyte with 50 $\mathrm{ppm}$ of water forms smooth and dense layer. The thickness of the highly ordered layer in Figure $1 \mathrm{~F}$ is $\sim 9 \mu \mathrm{m}$, which is close to the theoretical value of $8 \mu \mathrm{m}$ (based on capacity $1.5 \mathrm{mAh} \mathrm{cm}^{-2}$ used for Li deposition) and the diameter of $\mathrm{Li}$ nanorods of $\sim 260 \mathrm{~nm}$ growing perpendicular to the $\mathrm{Cu}$ substrate. We will also demonstrate an effect of the $\mathrm{LiNO}_{3}$ additive on Li-sulfur battery operation by operando ecTEM cell, which provides crucial insides into the performance of Li metal anodes and their successful incorporation into the next generation battery systems $[4,5]$.

\section{References:}

[1] X Ji, KT Lee and LF Nazar, Nat Mater 8 (2009), pp. 500-506.

[2] PG Bruce et al, Nat Mater 11 (2012), pp. 19-29.

[3] BL Mehdi et al, Nano Letters 15 (2015), p. 2168.

[4] J Qian et al, Nano Energy 15 (2015), pp. 135-144.

[5] This work was primarily supported by JCESR, an Energy Innovation Hub funded by DOE-BES. The development of the operando stage was supported by the Chemical Imaging LDRD Initiative at PNNL. PNNL is a multi-program national laboratory operated by Battelle for the U.S. DOE under Contract DEAC05-76RL01830. A portion of the research was performed at the EMSL user facility sponsored by DOE-BER and located at PNNL. The multi-target tracking algorithm is supported by NSF-1334012.
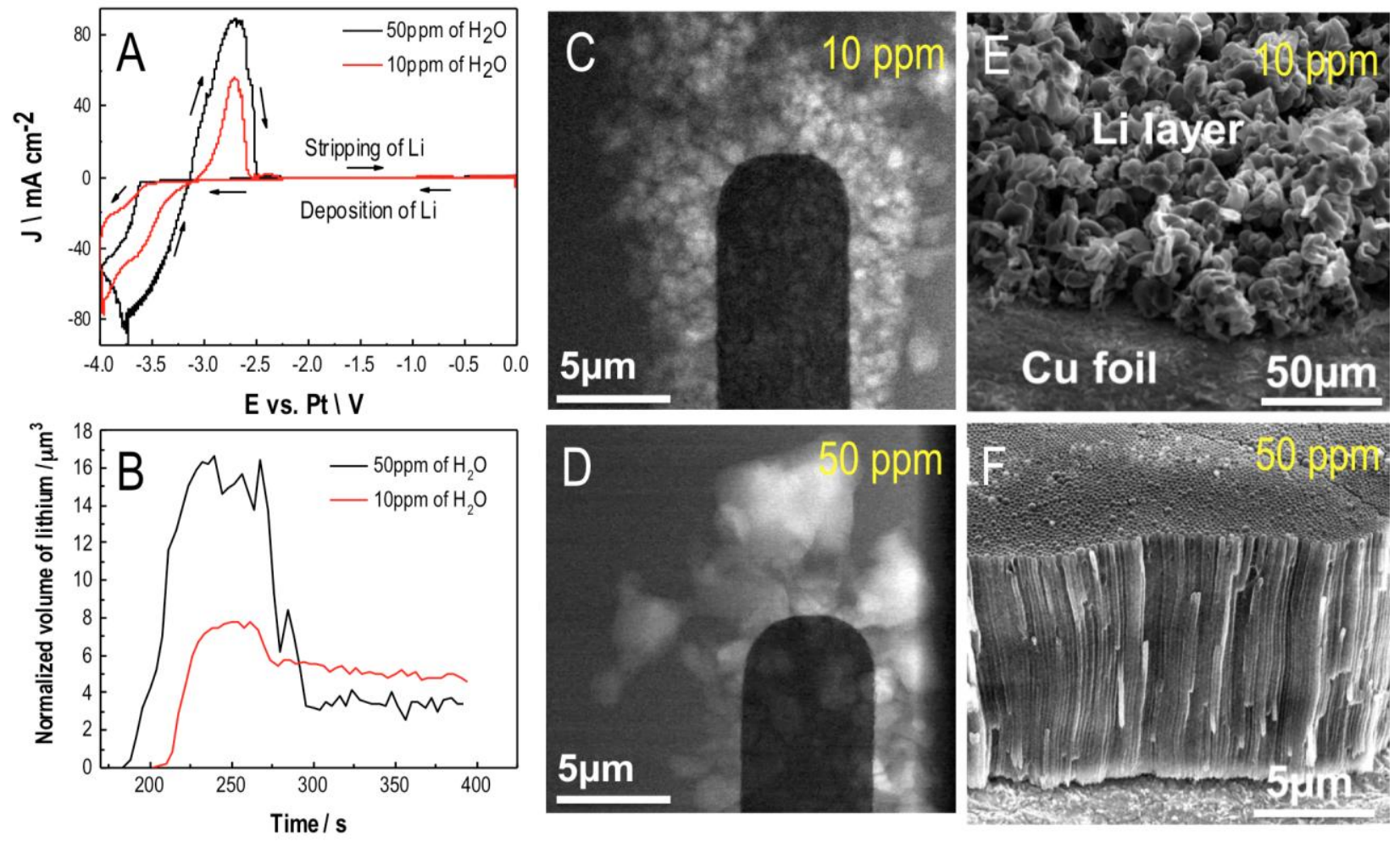

Figure 1. Cyclic voltammograms of the $\mathrm{LiPF}_{6} / \mathrm{PC}$ electrolyte with $10 \mathrm{ppm}$ (red) and $50 \mathrm{ppm}$ (black) of water (A) and quantified total area of Li deposited and stripped for the electrolyte with $10 \mathrm{ppm}$ (red) and 50ppm (black) of water. BF STEM images of Li deposits/grains at the interface between the Pt working electrode in the presence of $10 \mathrm{ppm}$ (C) and $50 \mathrm{ppm}$ (D) of water and corresponding SEM images for $10 \mathrm{ppm}(\mathrm{E})$ and 50ppm (F) of $\mathrm{Li}$ deposited at the $\mathrm{Cu}$ foil for $15 \mathrm{~h}$ at a $1 \mathrm{~mA} / \mathrm{cm}^{2}$ current densities. 